\title{
The effect of feeding a dairy-based calcium supplement on exercise-induced disturbance of bone turnover in healthy human subjects
}

\author{
R. Daniel and P. Jakeman \\ Human Science Research Unit, University of Limerick, Limerick, Republic of Ireland
}

Translating the current knowledge of nutrient and physical activity to an optimal prescription for bone health requires a better understanding of the interaction between diet and exercise in the maintenance of bone health. Nutrient support to bone health is well established and, although the optimal level of physical activity requires further definition, exercise is viewed as a positive benefit in the attainment and maintenance of peak bone mass ${ }^{(1)}$.

In a randomised placebo-controlled double-blind study the acute $(8 \mathrm{~h})$ change in serum C-terminal telopeptide of the $\alpha 1$ chain of type I collagen (CTX), a marker of bone resorption, was recorded following ingestion of a dairy-based Ca supplement $\left(1000 \mathrm{mg}\right.$; TruCal ${ }^{\mathrm{TM}}$; Glanbia Nutritionals Europe, Kilkenny, Republic of Ireland) in healthy human subjects (seven males and three females) at rest and following a lifestyle-related bout of physical activity ( $2 \mathrm{~h}$ treadmill walking; total energy expenditure $2 \mathrm{MJ}$ ). The study had the approval of the local research ethics committee.

Peak serum Ca occurred $3 \mathrm{~h}$ following ingestion, reducing the $8 \mathrm{~h}$ area under the curve (AUC) for serum parathyroid hormone by $20 \%$ and the $8 \mathrm{~h}$ AUC for serum CTX by $22 \%$ when compared with placebo. The effect of exercise was to increase serum CTX by approximately $10 \%$ in both the placebo and Ca trial, an effect that was not attributable to a change in plasma volume. The net treatment effect of $\mathrm{Ca}$ was to offset the exercise-induced increase in serum CTX, maintaining serum CTX $20 \%$ below basal levels achieved in the placebo trial at rest (Figure).

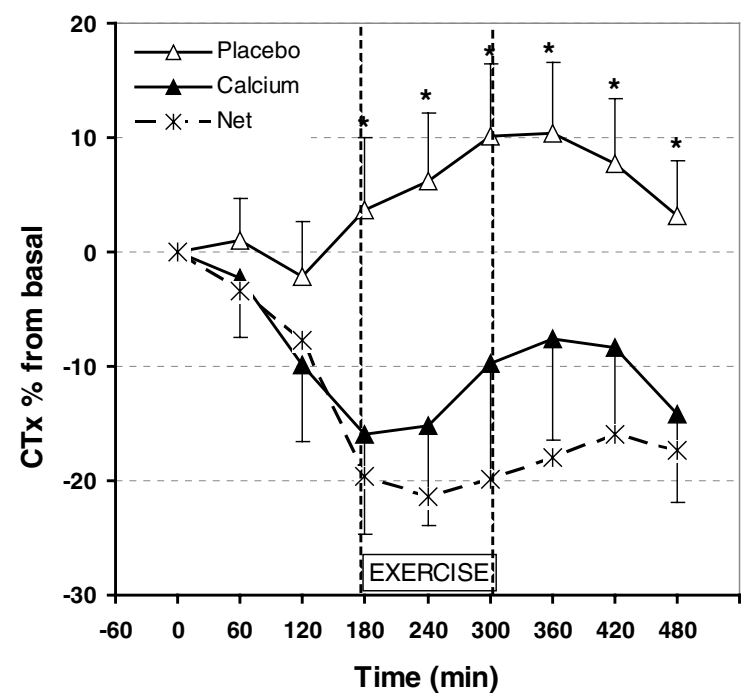

Measured by change in a biomarker of bone turnover, these data indicate an increase in bone resorption during and in the immediate period $(3 \mathrm{~h})$ post exercise. Corrected for diurnal variation, previous ingestion $(-3 \mathrm{~h})$ of $1000 \mathrm{mg}$ dairy Ca resulted in a significant reduction $(P<0.05$; ANOVA) in a biomarker of bone resorption, serum CTX. Dairy Ca did not affect the exercise-induced increase in serum CTX per se, but comfortably maintained the level of this biomarker of bone resorption below normal diurnal resting values.

The joint WHO/FAO expert consultation report has concluded that dietary and lifestyle recommendations developed for other chronic diseases may prove helpful to reduce osteoporotic risk ${ }^{(2)}$. These data suggest that the timing of supplemental Ca intake before exercise is an important consideration in the maintenance of bone health.

This research was funded by Enterprise Ireland and Glanbia Nutritionals.

1. Bailey CA \& Brooke-Wavell K (2008) Proc Nut Soc 67, 9-18.

2. World Health Organization/Food and Agriculture Organization (2003) Joint WHO/FAO Expert Consultation on Diet, Nutrition and the Prevention of Chronic Diseases. WHO Technical Report Series no. 916. Geneva: WHO. 